\title{
The Stanford stereotactic radiosurgery experience on 7000 patients over 2 decades (1999-2018): looking far beyond the scalpel
}

\author{
Nida Fatima, MBBS, MD, ${ }^{1}$ Antonio Meola, MD, PhD, ${ }^{1}$ Victoria Y. Ding, MS, ${ }^{1}$ Erqi Pollom, MD, MS, ${ }^{2}$ \\ Scott G. Soltys, MD, ${ }^{2}$ Cynthia F. Chuang, PhD, ${ }^{2}$ Nastaran Shahsavari, MD, ${ }^{1}$ \\ Steven L. Hancock, MD, ${ }^{2}$ Iris C. Gibbs, MD, ${ }^{2}$ John R. Adler, MD, ${ }^{1}$ and Steven D. Chang, MD ${ }^{1}$ \\ Departments of ${ }^{1}$ Neurosurgery and ${ }^{2}$ Radiation Oncology, Stanford University School of Medicine, Stanford, California
}

\begin{abstract}
OBJECTIVE The CyberKnife (CK) has emerged as an effective frameless and noninvasive method for treating a myriad of neurosurgical conditions. Here, the authors conducted an extensive retrospective analysis and review of the literature to elucidate the trend for CK use in the management paradigm for common neurosurgical diseases at their institution.

METHODS A literature review (January 1990-June 2019) and clinical review (January 1999-December 2018) were performed using, respectively, online research databases and the Stanford Research Repository of patients with intracranial and spinal lesions treated with CK at Stanford. For each disease considered, the coefficient of determination $\left(\mathrm{r}^{2}\right)$ was estimated as a measure of CK utilization over time. A change in treatment modality was assessed using a t-test, with statistical significance assessed at the 0.05 alpha level.
\end{abstract}

RESULTS In over 7000 patients treated with CK for various brain and spinal lesions over the past 20 years, a positive linear trend $\left(r^{2}=0.80\right)$ in the system's use was observed. CK gained prominence in the management of intracranial and spinal arteriovenous malformations (AVMs; $r^{2}=0.89$ and 0.95 , respectively); brain and spine metastases $\left(r^{2}=0.97\right.$ and 0.79 , respectively); benign tumors such as meningioma $\left(r^{2}=0.85\right)$, vestibular schwannoma $\left(r^{2}=0.76\right)$, and glomus jugulare tumor $\left(r^{2}=0.89\right)$; glioblastoma $\left(r^{2}=0.54\right)$; and trigeminal neuralgia $\left(r^{2}=0.81\right)$. A statistically significant difference in the change in treatment modality to CK was observed in the management of intracranial and spinal AVMs $(p<0.05)$, and while the treatment of brain and spine metastases, meningioma, and glioblastoma trended toward the use of CK, the change in treatment modality for these lesions was not statistically significant.

CONCLUSIONS Evidence suggests the robust use of CK for treating a wide range of neurological conditions.

https://thejns.org/doi/abs/10.3171/2020.9.JNS201484

KEYWORDS brain metastases; CyberKnife; glioblastoma; glomus jugulare tumors; intracranial; meningiomas; spine arteriovenous malformations; spine metastases; stereotactic radiosurgery; surgery; trigeminal neuralgia; vestibular schwannoma; vascular disorders

$\mathrm{T}$ HE CyberKnife (CK; Accuray Inc.) is a noninvasive frameless image-guided stereotactic radiosurgery (SRS) platform developed in 1994 by Stanford University-based neurosurgeon Dr. John R. Adler. Since then, CK has been used worldwide to treat a myriad of clinical conditions. At our institution, CK has been extensively used to treat numerous cancerous and noncancerous disorders of the nervous system. Although the effectiveness of and the indications for CK and other SRS techniques are already well established for some neurosurgical disorders, such as brain metastases, they are still debated for other diseases, such as arteriovenous malformation (AVM), and are under investigation for still other conditions, such as glioblastoma (GBM). Therefore, within this heterogeneous landscape, we performed a comprehensive review of our institutional data for some of the most common neurosurgical diseases amenable to SRS treatment, including AVM, meningioma, vestibular schwannoma, glomus jugulare tumor, brain metastases, spine metastases, GBM, and trigeminal neuralgia (TN). This study aims to elucidate the impact of and perspectives on CK SRS in the treatment paradigms for the abovementioned diseases through

ABBREVIATIONS $\mathrm{AVM}=$ arteriovenous malformation; $\mathrm{CDT}=$ Cohort Discovery Tool; $\mathrm{CK}=$ CyberKnife; $\mathrm{EBRT}=$ external beam radiation therapy; $\mathrm{EPIC}=\mathrm{Electronic}$ Privacy Information Center; GBM = glioblastoma; LINAC = linear accelerator; $n G B M=$ newly diagnosed GBM; $p G B M=$ progressive GBM; PPV = positive predictive value; SM = Spetzler-Martin; SRS = stereotactic radiosurgery; STARR = Stanford Research Repository; TN = trigeminal neuralgia.

SUBMITTED April 26, 2020. ACCEPTED September 11, 2020.

INCLUDE WHEN CITING Published online April 2, 2021; DOI: 10.3171/2020.9.JNS201484. 
an analysis of its frequency and trend of use over the past 2 decades, either as an alternative or as a complement to other therapeutic techniques. A systematic review of studies published by our institution on the use of SRS in the treatment of these diseases aims to highlight the current strengths and limitations of and future perspectives on CK SRS.

\section{Methods \\ Literature Review}

The PubMed, Embase, and Google Scholar databases were searched according to PRISMA guidelines ${ }^{1}$ using the Medical Subject Headings "CyberKnife," "arteriovenous malformations," "benign intracranial tumors," "meningioma," "vestibular schwannoma," "glomus jugulare tumor," "malignant brain tumor," "malignant spinal cord tumor," "brain metastases," "spine metastases," "glioblastoma," and "trigeminal neuralgia" for the period from January 1990 through June 2019. All articles that reported on the use of CK in treating intracranial and spinal lesions at our institution were included in our study. Studies that reported on radiation therapy and alternative SRS techniques (Gamma Knife, Elekta AB; intensity-modulated radiation therapy) were excluded from consideration. Two reviewers (N.F. and A.M.) extracted data from each article by using a structured template provided by the Cochrane Consumers and Communication Group. These items included 1) demographic characteristics, 2) clinical characteristics, 3) target volume $\left.\left(\mathrm{cm}^{3}\right), 4\right)$ median prescribed dose (Gy), 5) conformity index, 6) number of fractions, 7) median follow-up (months), 8) tumor size at last follow-up, 9) tumor control rate, 10) symptomatic control rate, and 11) complications. Any disagreement between the reviewers was resolved by discussion.

\section{Clinical Data Review}

Clinical data on all Stanford patients treated with CK between January 1999 and December 2018 are maintained in an institutional review board-approved database. We conducted our retrospective search using the Cohort Discovery Tool (CDT) available through the Stanford Research Repository (STARR). The search terms "CyberKnife," "surgery," "endovascular," "intracranial lesions," "spinal lesions," "arteriovenous malformations (AVMs)," "meningioma," "vestibular schwannoma," "glomus jugulare tumor," "brain metastases," "spine metastases," "glioblastoma," and "trigeminal neuralgia" in combination with the Boolean operators "and/or" were used to identify patient cohorts. AVMs were classified using the Spetzler-Martin (SM) grading system and then grouped into grades I and II, grade III, and grades IV and V.

The total number of patients with intracranial and spinal lesions treated with CK were binned into the following 5-year intervals to compare the utilization of different treatment techniques across different time intervals: 1999-2003, 2004-2008, 2009-2013, and 2014-2018.

\section{Statistical Analysis}

Patient counts, stratified by treatment modality (surgery, endovascular, CK, or multimodal), were aggregated and plotted across the abovementioned 5-year intervals for the following nine pathologies: intracranial (categorized by SM grade) and spinal AVMs, brain and spine metastases, meningioma, vestibular schwannoma, glomus jugulare tumor, GBM, and TN. A linear regression line was fitted to each CK trajectory, and the corresponding coefficient of determination $\left(r^{2}\right)$ was estimated as a measure of utilization.

A Student t-test was used to assess change in patient count over time for each treatment modality, with statistical significance assessed at the 0.05 alpha level. In addition, stacked bar charts were constructed to show the percentage of patients undergoing each type of treatment modality over time in order to determine if and how the treatment paradigm for different diseases changed over the past 2 decades.

We also determined the positive predictive value (PPV) of our STARR search by comparing the cohort size identified via the CDT to the number of patients who had undergone the searched treatment modality according to the Stanford Health Care Electronic Privacy Information Center (EPIC) electronic medical records.

All analyses were performed using Microsoft Excel and IBM SPSS Statistics software v23.0 (IBM Corp.).

\section{Treatment Fractionation}

For each of the three most frequent (50 cases/time frame, minimum) neurosurgical diseases treated at our CK center, namely brain metastases, meningioma, and vestibular schwannoma, the number of fractions was extracted from 50 randomly selected charts from each of the three most recent time frames (2004-2008, 2009-2013, 2014-2018). The number of fractions was inconsistently reported in the time frame 1999-2003 and was not taken into consideration. The Kruskal-Wallis test (the nonparametric alternative to the one-way ANOVA) was used to assess the distribution of the number of fractions over time.

\section{Results}

\section{Literature Review}

A total of 2500 articles were retrieved from electronic databases and reviewed according to PRISMA guidelines. ${ }^{1}$ After removing duplicate search results, 1388 articles were excluded because their content was unrelated to $\mathrm{CK}$, and 572 articles were further excluded on the basis of eligibility criteria, leaving 40 articles for qualitative review and 31 full-text articles for the final quantitative review (Fig. 1).

\section{Overview of Clinical Applications and Paradigm Shift}

Our institution has treated over 7000 patients with CK over the past 2 decades, including those with benign brain tumors (meningiomas, vestibular schwannomas, glomus jugulare tumors, nonvestibular schwannomas, chordomas, hemangioblastomas, and ependymomas), AVMs (intracranial and spinal cord AVMs), malignant tumors (brain and spine metastases, chondrosarcomas, and GBM), and resection cavities of brain metastases. A positive linear trend $\left(r^{2}=0.80\right)$ in the system's use was observed. The next sections focus on the most frequently treated neurosurgical 


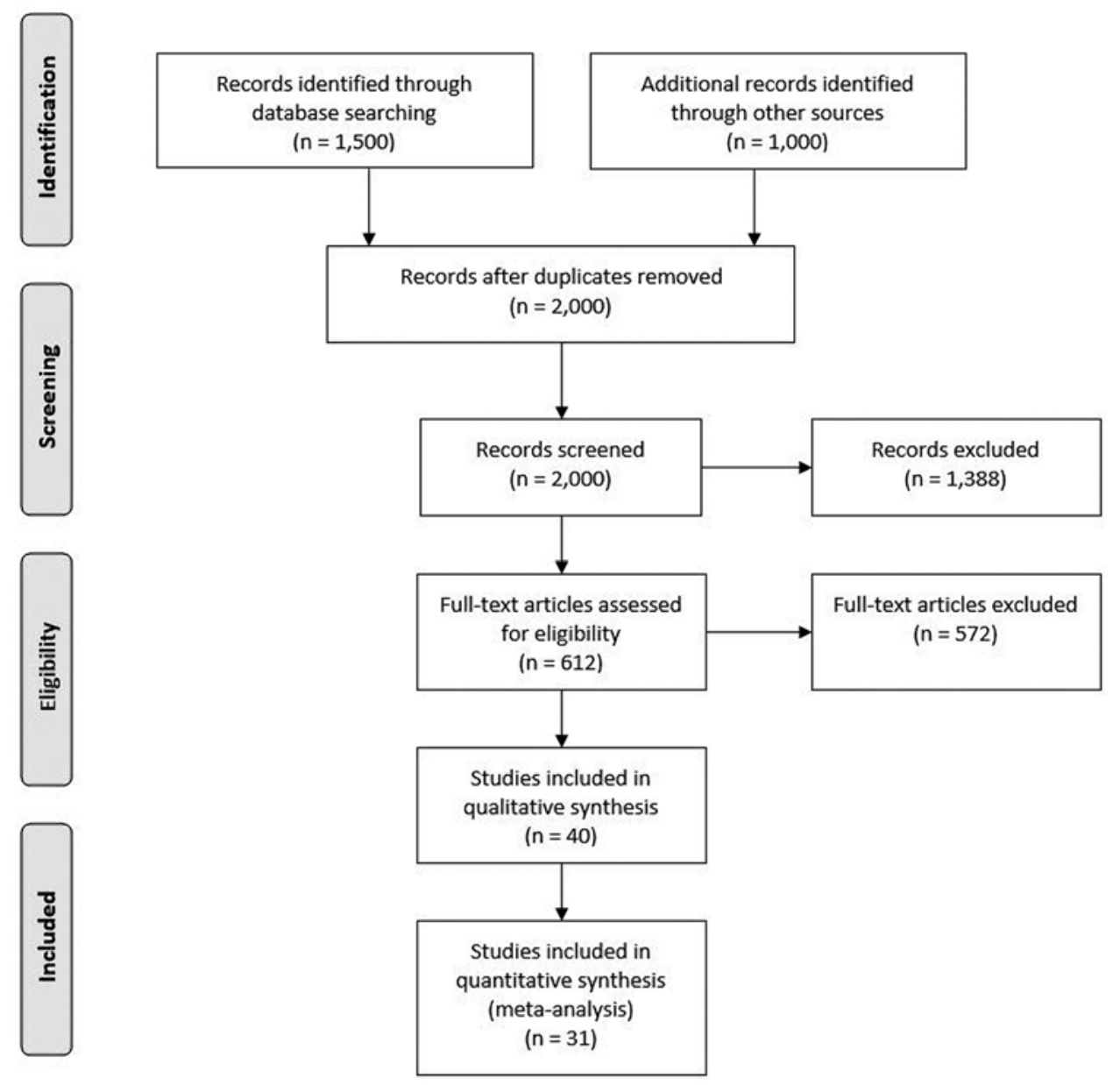

FIG. 1. An electronic database search of Stanford studies according to PRISMA guidelines. Data added to the PRISMA template (from Moher D, Liberati A, Tetzlaff J, Altman DG, The PRISMA Group (2009). Preferred Reporting Items for Systematic Reviews and Meta-Analyses: The PRISMA Statement. PLoS Med 6[7]: e1000097) under the terms of the Creative Commons Attribution License.

diseases at our institution. Note that $\mathrm{CK}$ was also used to treat cluster headache and facetogenic back pain, which are considered new frontiers for this technology.

For each pathology considered, the PPV was calculated as the proportion of subjects whose pathology had been confirmed via EPIC chart review among all subjects identified by the CDT to have the pathology. We then reported the average PPV, which was $84.8 \%$. On the other hand, cases might have been missed, but since EPIC verification was not performed for subjects not identified by the CDT, we could not calculate how many cases were not documented in a way that could be detected by the CDT. Below we summarize the use of CK for the most common diseases amenable to SRS.

\section{Intracranial and Spinal AVMs}

AVMs pose a definite surgical challenge because of their location either in eloquent areas or close to nearby critical neurovascular structures. SRS has extensively influenced the management of intracranial and spinal cord AVMs. Depending on the patient and AVM characteris- tics, SRS can be used as an alternative or an adjuvant to endovascular embolization and microsurgical resection. The rationale for SRS is that an adequate radiation dose causes gradual narrowing and potential obliteration of the vascular lumen over a period of 2 to 3 years. ${ }^{2}$

Our analysis showed that there was a statistically significant increase in the frequency of using CK alone $\left(\mathrm{r}^{2}\right.$ $=0.89, \mathrm{p}=0.05$ ) for the treatment of intracranial AVMs (Fig. 2A-I). Although the overall percentage of cases treated with CK alone remained grossly stable (10.3\% to $7.9 \%$ ) across the past 20 years, the percentage of cases treated with a combination of treatments (which at least in part includes CK) increased from $12.8 \%$ in 1999-2003 to $26.4 \%$ in 2014-2018. Further dichotomization based on the grading of AVMs confirmed that, although combined treatment modalities (surgery, endovascular, and/or CK) remained the treatment of choice, there was a positive linear trend in CK use (SM grades I and II, $\mathrm{r}^{2}=0.79$; SM grade III, $\mathrm{r}^{2}=0.83$; $\mathrm{SM}$ grades IV and $\mathrm{V}, \mathrm{r}^{2}=0.93$ ), as shown in Fig. 2A-I. In a comparative analysis of the treatment modalities based on SM grades in the last 4 years (2014-2018), the higher the SM grade, the more frequently 

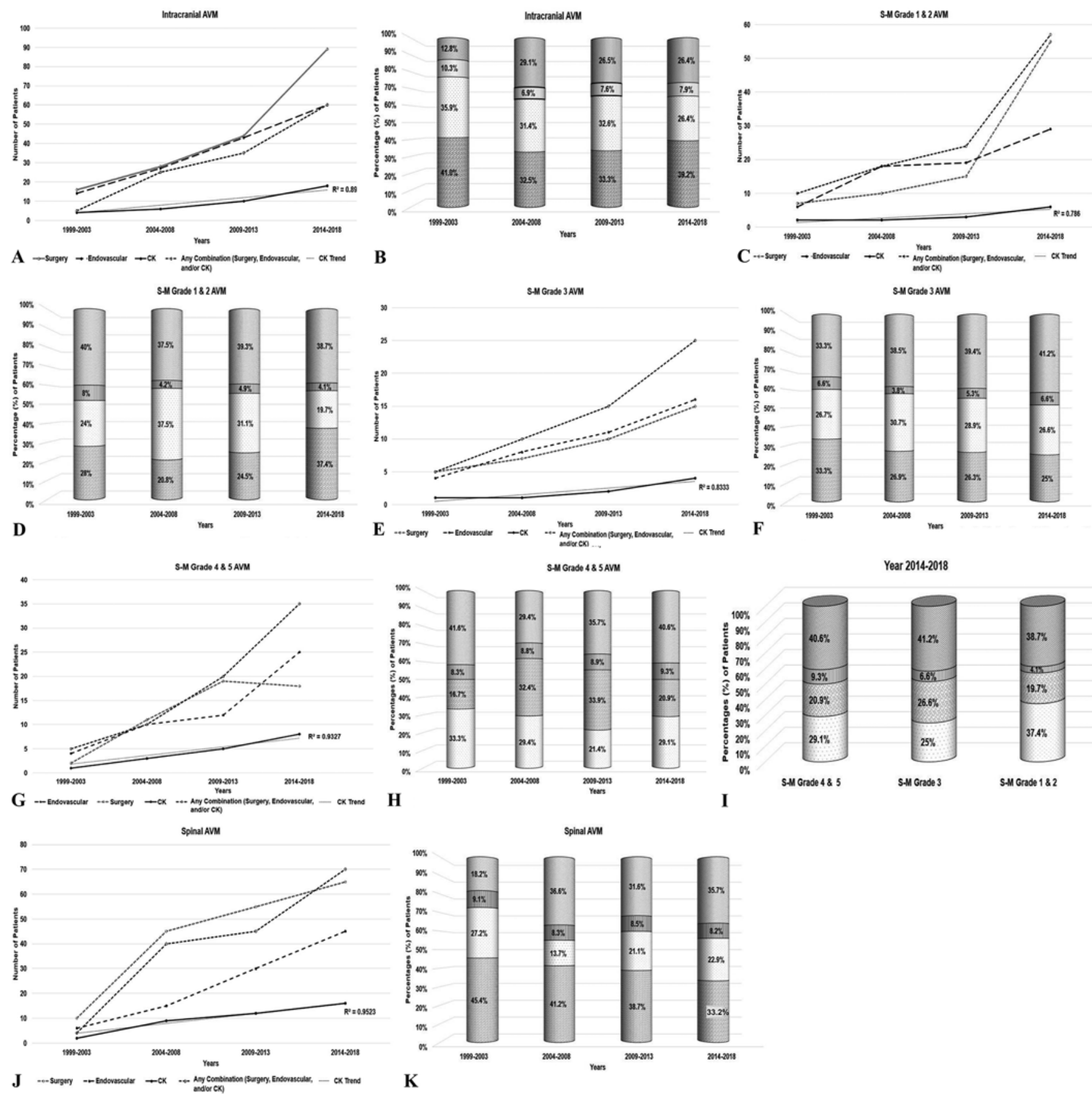

K

FIG. 2. A: Number (A) and percentage (B; each bar from top to bottom represents combination therapy [surgery, endovascular therapy, and/or CK], CK, endovascular therapy, and surgery) of patients with intracranial AVMs treated with surgery, CK radiosurgery, endovascular treatment, and any combination of the three techniques in consecutive 5-year time frames over the past 20 years. Number (C) and percentage (D; each bar from top to bottom represents combination therapy [surgery, endovascular therapy, and/or CK], CK, endovascular therapy, and surgery) of patients with SM grade I and II intracranial AVMs treated with surgery, CK radiosurgery, endovascular treatment, and any combination of the three techniques in consecutive 5-year time frames over the past 20 years. Number $(\mathbf{E})$ and percentage ( $F$; each bar from top to bottom represents combination therapy [surgery, endovascular therapy, and/or CK], CK, endovascular therapy, and surgery) of patients with SM grade III intracranial AVMs treated with surgery, CK radiosurgery, endovascular treatment, and any combination of the three techniques in consecutive 5-year time frames over the past 20 years. Number (G) and percentage $(\mathrm{H}$; each bar from top to bottom represents combination therapy [surgery, endovascular therapy, and/or CK], CK, surgery, and endovascular therapy) of patients with SM grade IV and V intracranial AVMs treated with surgery, CK radiosurgery, endovascular treatment, and any combination of the three techniques in consecutive 5-year time frames over the past 20 years. Percentage of patients (I; each bar from top to bottom represents combination therapy [surgery, endovascular therapy, and/or CK], CK, surgery, and endovascular therapy) with SM grade I and II, grade III, and grade IV and $\mathrm{V}$ intracranial AVMs treated with surgery, CK radiosurgery, endovascular treatment, and any combination of the three techniques over the past 5 years (2014-2018). Number (J) and percentage (K; each bar from top to bottom represents combination therapy [surgery, endovascular therapy, and/or CK], $\mathrm{CK}$, endovascular therapy, and surgery) of patients with spinal AVMs treated with surgery, CK radiosurgery, endovascular treatment, and their combination in consecutive 5 -year time frames over the past 20 years. 
CK alone was used $(\mathrm{p}=0.05)$ as well as combined modalities $(\mathrm{p}<0.001)$ (Supplemental Tables $1-5)$. Our data are consistent with those from previous series ${ }^{3}$ showing that, in selected patients harboring high-grade AVMs, SRS can be performed alone or in combination with other treatment techniques in order to minimize the morbidity related to surgery and endovascular treatment. Moreover, in our experience, ${ }^{2}$ complex AVMs that had failed to obliterate 4 years after initial treatment were successfully obliterated in two-thirds of cases at 84 months after CK treatment (Table 1).

Although endovascular treatment and resection alone or in combination are often the treatment of choice in spinal AVMs, the associated risk of morbidity prompted the use of SRS as primary or adjuvant treatment for these lesions (Fig. 2J-K). We observed a statistically significant linear trend toward the use of CK $\left(r^{2}=0.95, \mathrm{p}=0.04\right.$; Supplemental Table 6). Interestingly, across the past 20 years, $8.2 \%$ to $9.1 \%$ of spinal AVMs were treated with SRS, while $18.2 \%$ to $35.7 \%$ required a multimodal treatment.

In our institutional experience, at a median follow-up of 27.9 months (range 16-54 months), symptomatic control was observed in a median of $92.3 \%$ (range $86.0 \%-$ $100.0 \%)^{4-8}$ and complete obliteration of the nidus occurred in a median of $13.2 \%$ (range $6.6 \%-26.6 \%)^{4-8}$ of the patients with spinal cord AVMs following CK as either primary treatment or adjuvant treatment to embolization, radiosurgery, and/or microsurgical resection (Table 1).

Although surgery and endovascular treatment remain the mainstays of treatment for brain and spinal AVMs, SRS is emerging as an adjuvant or stand-alone therapy, especially in cases of high-grade brain AVMs and spinal AVMs, for which there is still a special need for innovative and minimally invasive treatments given the high surgical morbidity.

\section{Brain and Spine Metastases}

CK is now being considered the treatment of choice for patients with brain metastases, with a tumor control rate of $85 \%-95 \% .^{9}$ With the exception of selected cases requiring surgery, SRS is currently used as stand-alone treatment for patients with a single metastasis, oligometastatic disease, or multimetastatic disease ( $>4$ metastases).${ }^{10}$ When surgery is required, postoperative SRS to the resection cavity of the metastasis is highly recommended in order to reduce the risk of local recurrence..$^{11}$ According to this evidence and our analysis, there was a positive linear trend toward the treatment of brain metastases using CK $\left(\mathrm{r}^{2}=\right.$ 0.97, $\mathrm{p}=0.14$; Supplemental Table 7). Moreover, between 2014 and 2018, almost all patients with brain metastases (99.7\%) underwent SRS or a combination of surgery and SRS (Fig. 3A-B). In our institutional analysis, the pooled estimate of local tumor control was a median of $84 \%$ (range $79.2 \%-100 \%)^{9,12-15}$ at a median clinical follow-up of 10.5 months (range 5-18 months) ${ }^{9,12-15}$ after CK for brain metastases (Table 2).

Spine metastases are historically treated with surgery, which can be followed by conventional external beam radiation therapy (EBRT), to provide pain relief and prevent further tumor growth and vertebral body collapse..$^{16}$ At our institution, surgery alone or in combination with EBRT is still the treatment of choice. However, SRS is used alone or in combination with surgery in a growing number of cases, that is, from $8.3 \%$ in $1999-2003$ to $27.2 \%$ in 2014 2018. In the future, SRS could, at least in part, replace the role of EBRT for spine metastases. In our experience, clinical improvement was observed in a median of $51.9 \%$ (range $20 \%-83.8 \%)^{17,18}$ of the patients and local tumor control was obtained in $100 \%{ }^{18}$ of patients after CK treatment for spinal metastases (Fig. 3C-D, Table 2, Supplemental Table 8).

\section{Benign Intracranial Tumors}

SRS is now extensively used for the treatment of benign intracranial tumors, and at our institution, CK showed a positive linear trend for the treatment of patients with intracranial meningioma $\left(r^{2}=0.85, p=0.11\right.$; Fig. 3E-F, Supplemental Table 9), vestibular schwannoma $\left(r^{2}=0.76\right.$, $\mathrm{p}=0.14$; Fig. 3G-H, Supplemental Table 10), and glomus jugulare tumor $\left(r^{2}=0.89, p=0.07\right.$; Fig. 3I-J, Supplemental Table 11). SRS is an ideal treatment for these slowgrowing, noninvasive tumors, which can take months to years to shrink after radiation. According to the linear quadratic model of radiobiology, fewer radiation fractions yield greater potency, which could explain the treatment effectiveness of SRS on these tumors (Table 3).

For meningiomas, surgery remains the main treatment option, although CK as primary or adjuvant treatment was increasingly used, from $8 \%$ in $1999-2003$ to $41.7 \%$ in 2014-2018. At our institution, pooled local tumor control was $93 \%$ after treatment with SRS for benign intracranial tumors. ${ }^{19-25}$ The indications for CK for meningiomas became progressively broader, including giant meningiomas, ${ }^{25}$ atypical and malignant meningiomas, ${ }^{24}$ and meningiomas close to critical neurovascular structures, such as perioptic meningiomas. ${ }^{20}$

SRS revolutionized the treatment paradigm for vestibular schwannomas, which in 1999-2003 were mainly treated with surgery only (75\%) and are now (2014-2018) mainly treated with CK alone (68.3\%). In our series, local tumor control after CK was $97 \%$ for vestibular schwannomas. ${ }^{26}$

As with vestibular schwannomas, SRS impacted the treatment paradigm for head and neck paragangliomas, which in 1999-2003 were mainly treated with surgery only (81.8\%) and more recently (2014-2018) are mainly treated with $\mathrm{CK}$ alone $(60 \%)$. In our series, local tumor control was $100 \%{ }^{27,28,47}$ after CK treatment.

\section{Glioblastoma}

Resection followed by adjuvant radiochemotherapy is the treatment of choice for GBM, ${ }^{30}$ leading to a mean survival of around 9-14 months. SRS could be offered as a palliative treatment in selected cases, although the benefit in overall survival is still unclear. At our institution, although the use of CK has a positive trend $\left(r^{2}=0.54, p\right.$ $=0.02$ ), it is still used only as an adjuvant treatment in $12.5 \%$ of patients in 2014-2018 (Fig. 3K-L, Supplemental Table 12).

More than a decade ago, our group published 2 multicentric studies about the use of CK SRS for GBM. Lipani 


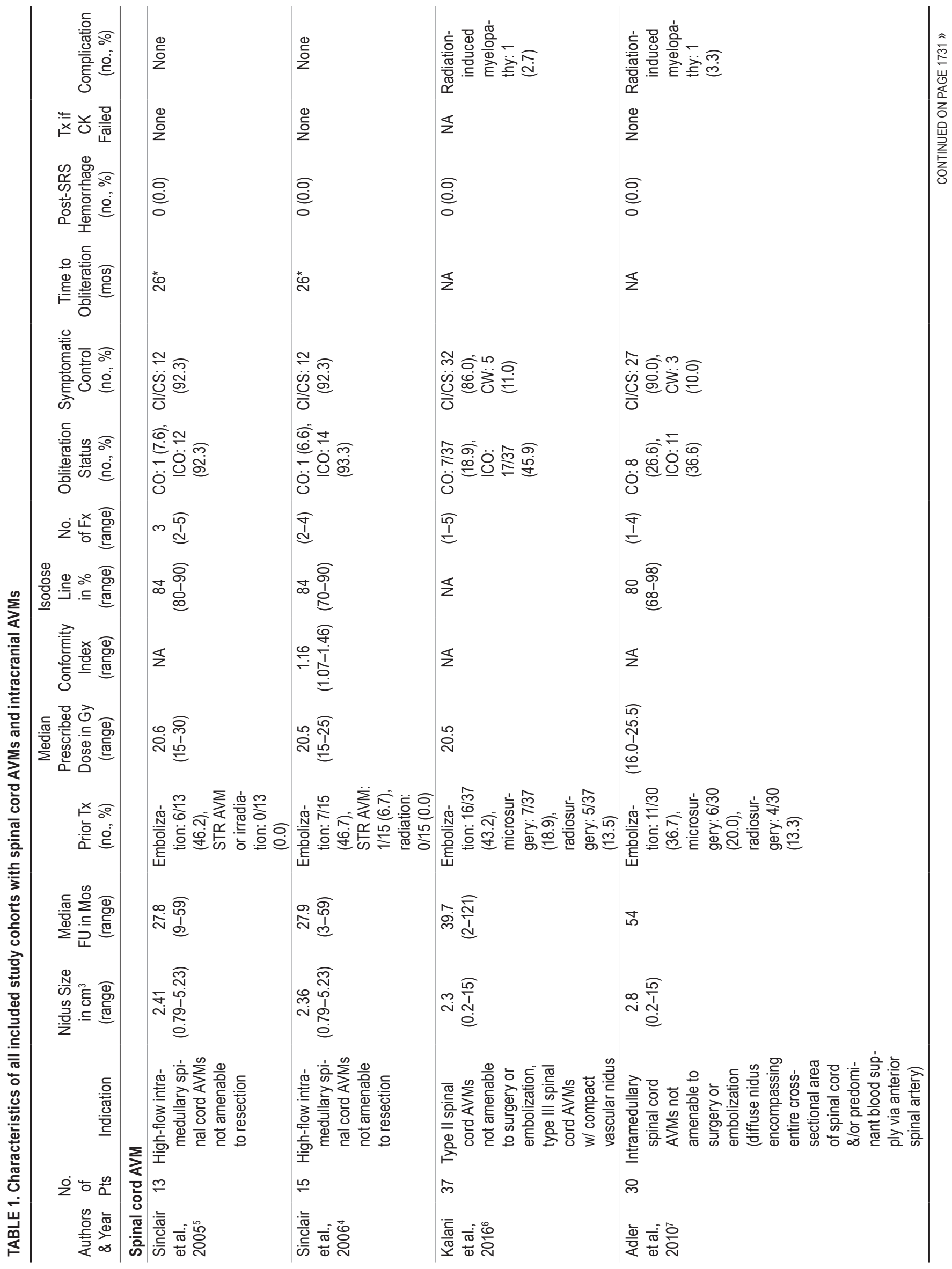




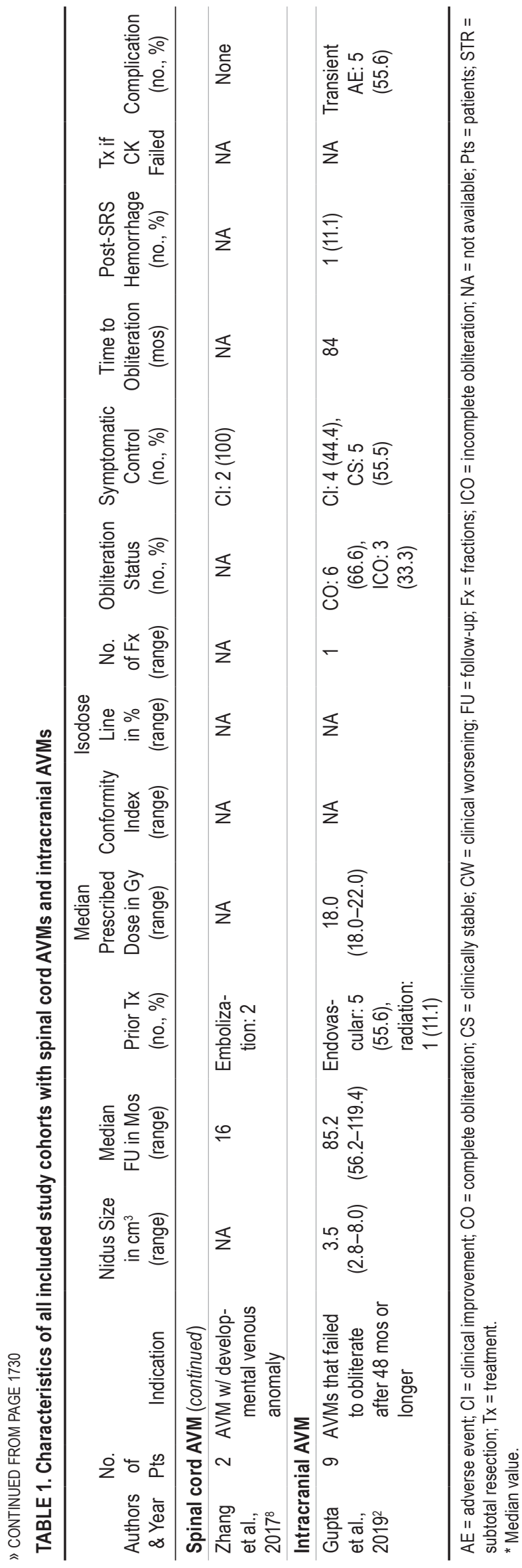

et al. ${ }^{31}$ presented a cohort of 20 newly diagnosed GBMs (nGBMs) treated with postsurgical hypofractionated SRS between 2000 and 2004. Surgery consisted of grosstotal resection, subtotal resection, or biopsy. Patients did not receive any other form of radiation besides CK SRS. Eight patients received adjuvant chemotherapy (nimustine [ACNU] or vincristine). A mean marginal dose of 34.58 Gy (range 19.99-41.47 Gy) with a mean isodose line of $79.25 \%$ (range $50.38 \%-85.68 \%$ ) was delivered via a mean of 5.65 fractions (range 1-8 fractions) to a mean tumor volume of $86.98 \mathrm{~cm}^{3}$ (range $9.62-185.81 \mathrm{~cm}^{3}$ ). Data about tumor markers were not available. The median survival was 16 months, while the 2-year survival was $33.8 \%$.

In the study by Villavicencio et al., ${ }^{32}$ a total of 46 patients with either nGBM or progressive GBM (pGBM) were treated between 2002 and 2005 . The nGBM group included 20 (43.5\%) patients receiving CK SRS either as the primary treatment or as a radiosurgical boost shortly after surgery or surgery and standard EBRT. A median margin dose of 20 Gy (range 12-25 Gy) was delivered via a median of 1 fraction (range 1-5 fractions) to a median target volume of $5.8 \mathrm{~cm}^{3}$ (range $0.7-47.3 \mathrm{~cm}^{3}$ ) with a median isodose of $74.9 \%$ (range $66.1 \%-89.0 \%$ ). The pGBM group included $26(56.5 \%)$ patients treated at the time of tumor recurrence or progression. A median margin dose of 20 Gy (range 8-25 Gy) was delivered via a median of 2 fractions (1-5) to a median target volume on $7 \mathrm{~cm}^{3}$ (range $\left.0.4-48.5 \mathrm{~cm}^{3}\right)$ with a median isodose of $77.7 \%(65.0 \%-$ $88.0 \%)$.

Overall, the only statistically significant differences between the nGBM and the pGBM groups were the higher mean EBRT dose and the lower average recursive partitioning analysis class in the pGBM group. EBRT was performed in $75 \%$ of nGBM cases and $100 \%$ of pGBM cases, whereas chemotherapy was administered in $75 \%$ of nGBM cases and $96 \%$ of pGBM cases. Data about tumor markers were not available.

The median survival from diagnosis for the nGBM group was 11.5 months (range 2-33 months) compared to 21 months (range 8-96 months) for the pGBM group. This difference was statistically significant (Kaplan-Meier analysis, $\mathrm{p}=0.0004$ ). The median survival times from the CK SRS were 9.5 months (range 0.25-31 months) and 7 months (range 1-34 months) for the nGBM and pGBM groups (Kaplan-Meier analysis, $\mathrm{p}=0.79$ ), respectively.

Further studies are warranted to investigate the impact of $\mathrm{CK}$ in the treatment of primary and recurrent GBM (Table 4).

\section{Trigeminal Neuralgia}

Although microvascular decompression has an excellent success rate for treating classic TN due to neurovascular conflict, SRS emerged as a useful tool for the treatment of cases not amenable to surgery, such as atypical $\mathrm{TN}$, or for recurrent TN after surgery. Our institutional review confirmed that, while the majority of patients were successfully treated with surgery, CK alone has been used more frequently in terms of the number of patients $\left(\mathrm{r}^{2}=\right.$ $0.80, p=0.11$ ) (Supplemental Table 13). However, its utilization compared to surgery declined from $38.5 \%$ in $1999-$ 2003 to $19.4 \%$ in $2014-2018$ (Fig. 4). 


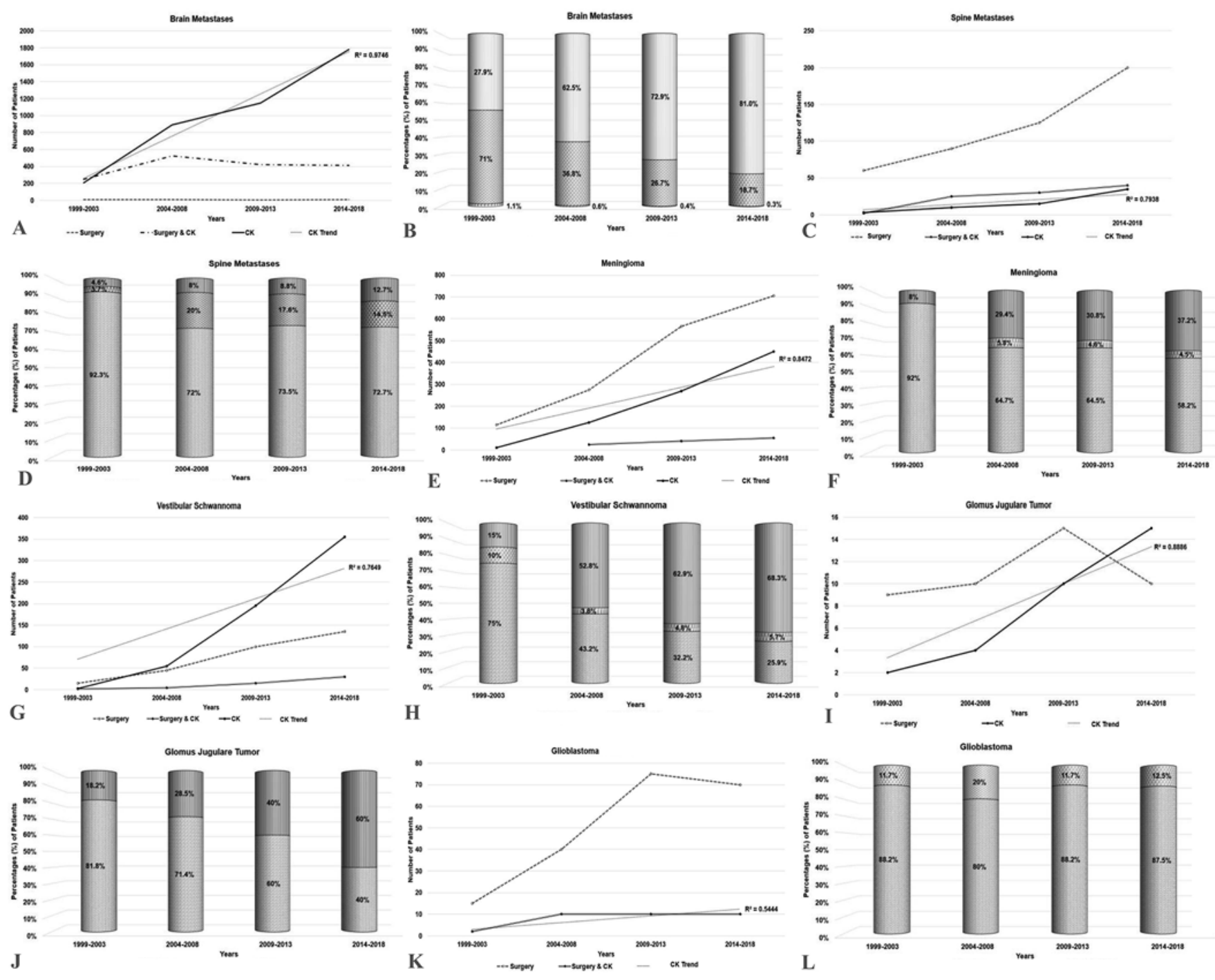

FIG. 3. Number of patients with brain metastases (A), spine metastases (C), meningiomas (E), vestibular schwannomas (G), glomus jugulare tumors (I), or GBMs (K) treated with surgery, CK radiosurgery, or their combination in consecutive 5-year time frames over the past 20 years. Percentage of patients with brain metastases (B; each bar from top to bottom represents CK, surgery plus $\mathrm{CK}$, and surgery), spine metastases (D; each bar from top to bottom represents CK, surgery plus CK, and surgery), meningiomas (F; each bar from top to bottom represents CK, surgery plus CK, and surgery), vestibular schwannomas (H; each bar from top to bottom represents CK, surgery plus CK, and surgery), glomus jugulare tumors (J; each bar from top to bottom represents CK and surgery), or GBMs (L; each bar from top to bottom represents CK plus surgery and surgery) treated with each treatment modality or combination in each 5 -year period.

With a median follow-up of 10.75 months (range 4-25 months), clinical improvement was observed in $85.7 \%$ (range 70\%-97.8\%) and symptoms recurred in a median of $16.0 \%$ (range $6.9 \%-42.9 \%$ ) of the patients following CK treatment of typical TN (Table 5). ${ }^{29,33-38,51}$ Only 1 included study performed CK for the management of atypical TN, ${ }^{35}$ and clinical improvement was observed in $85.7 \%$ of the patients with a recurrence in $42.9 \%$ of the patients.

\section{Treatment Fractionation}

For brain metastases, the median numbers of fractions (IQR) in the three most recent time frames (2004-2008, 2009-2013, 2014-2018) were 1.0 (1.0-2.0), 2.0 (1.0-3.0), and $1.5(1.0-3.0)$, respectively $(\mathrm{p}=0.097)$. For meningiomas, the median numbers of fractions (IQR) in the three most recent time frames were $2.0(1.0-3.0), 3.0$ (1.0-3.0), and $3.0(1.0-3.0)$, respectively $(\mathrm{p}=0.171)$. Thus, for both brain metastases and meningiomas, our data suggest a nonsignificant expansion in the number of fractions over time, comparing the 2004-2008 time frame with the two subsequent time frames.

Finally, for vestibular schwannomas, the median number of fractions (IQR) remained 3.0 (3.0-3.0) across all time frames $(p=0.021)$. However, the percentage of vestibular schwannomas treated with 3 fractions declined from $98 \%$ in $2004-2008$ to $96 \%$ in $2009-2013$ to $82 \%$ in 2014-2018, in favor of shorter fractionation. 


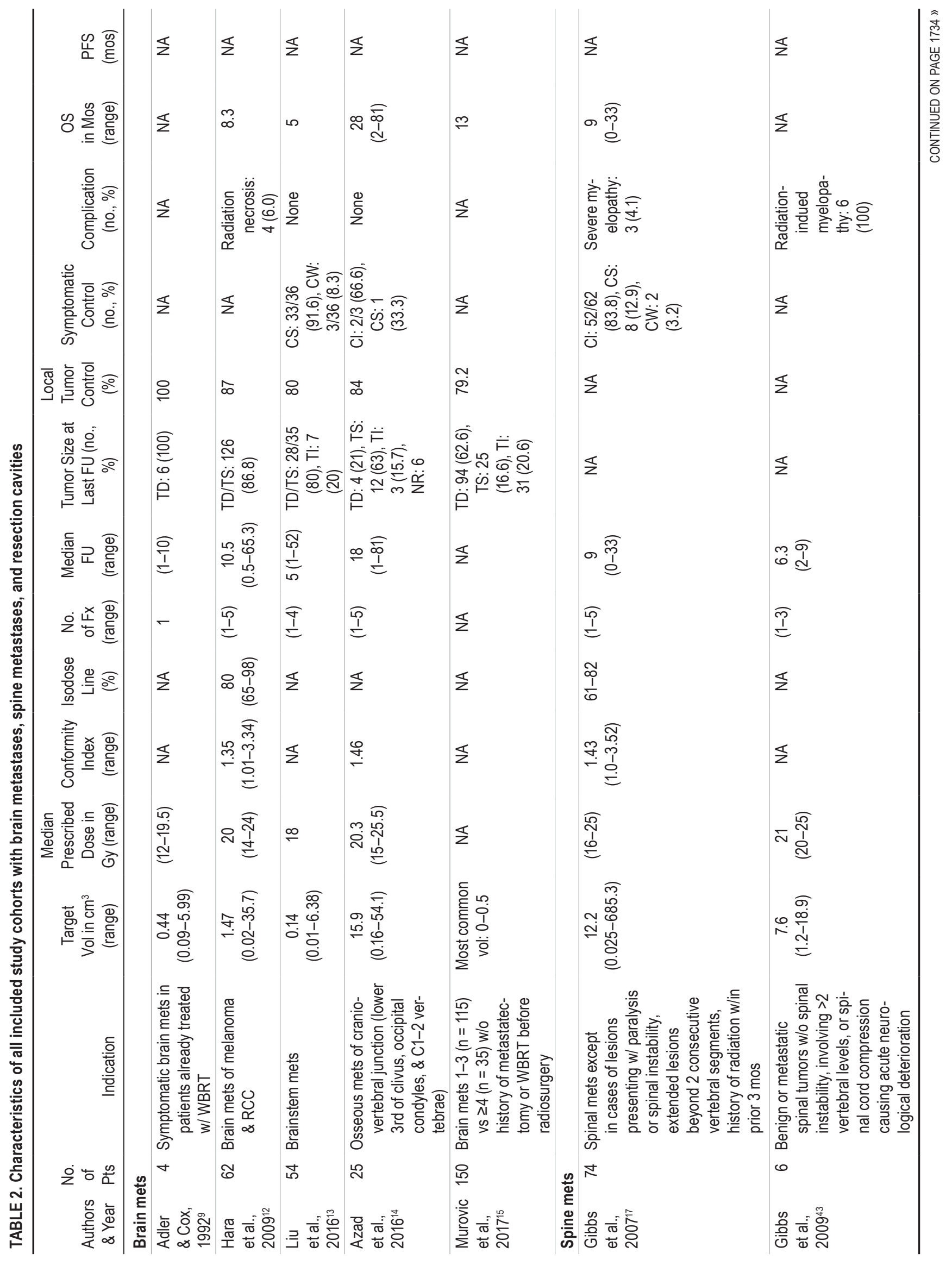




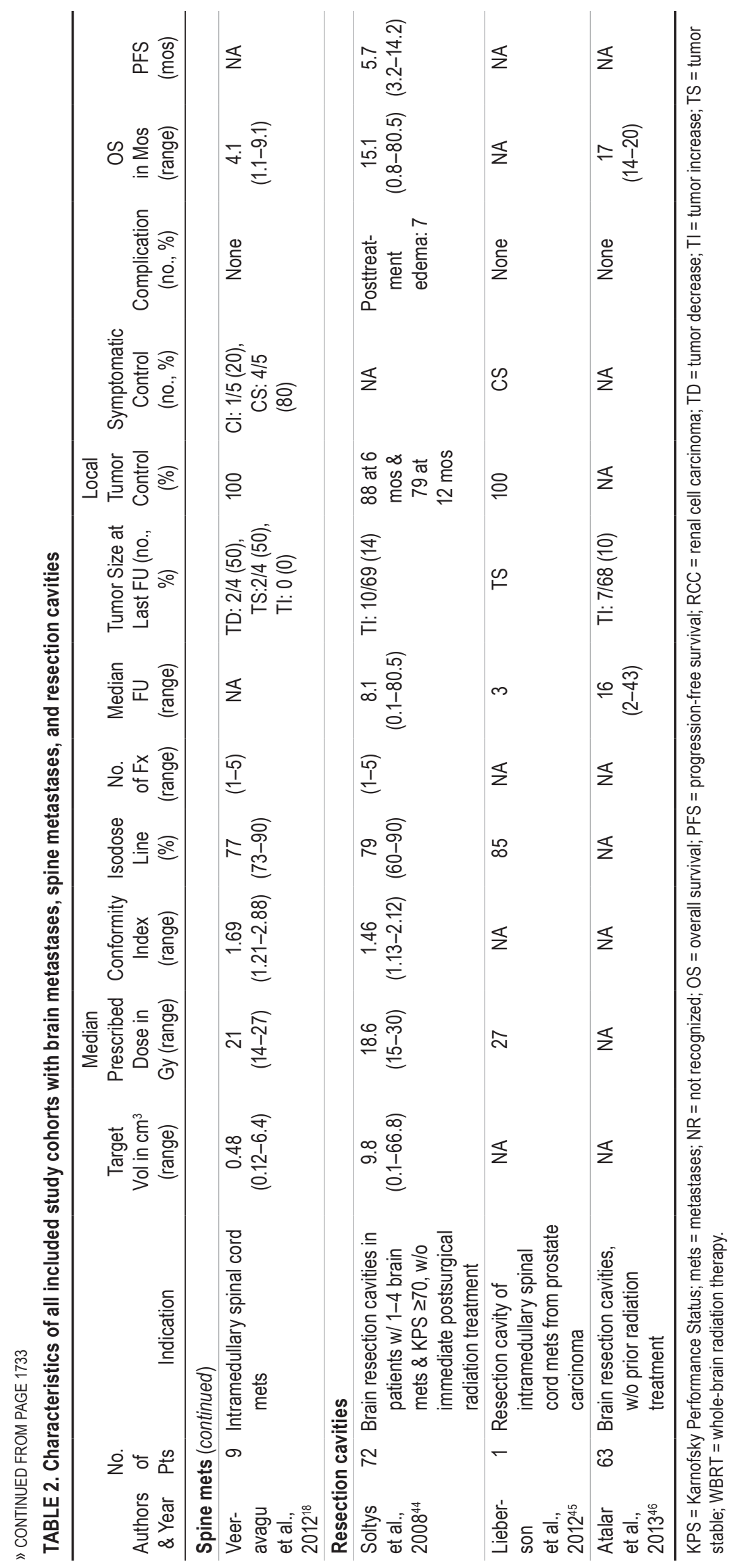




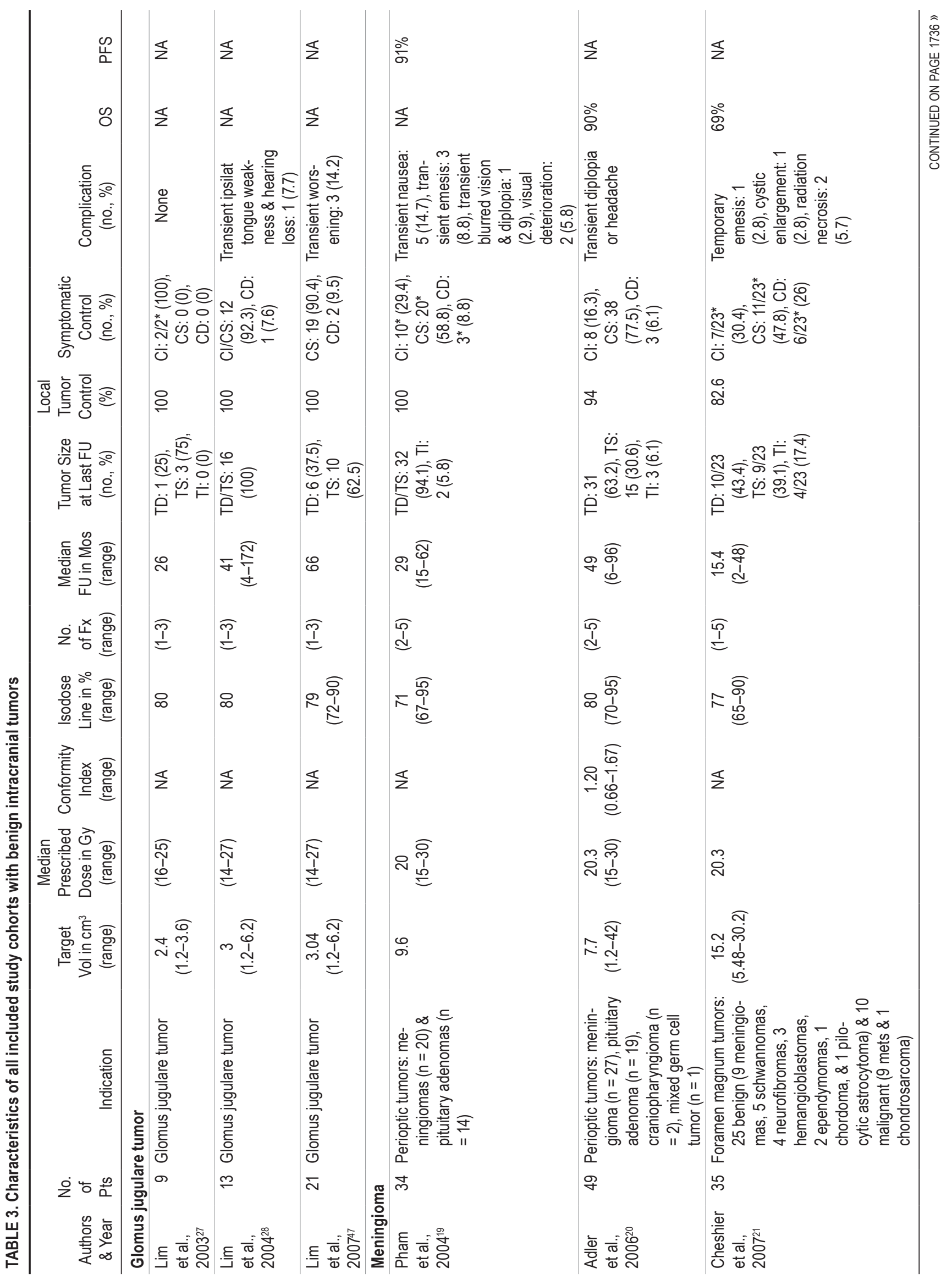




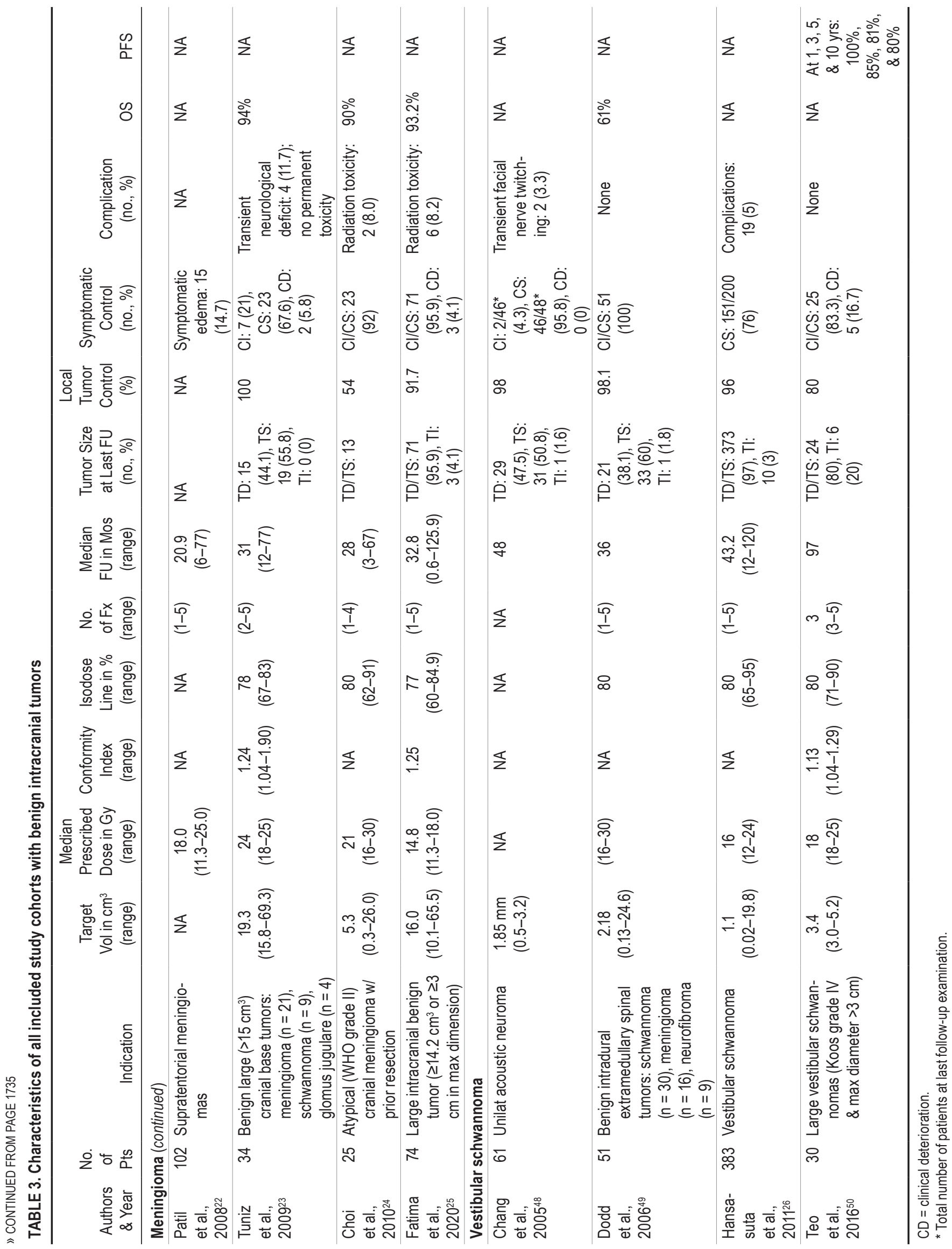




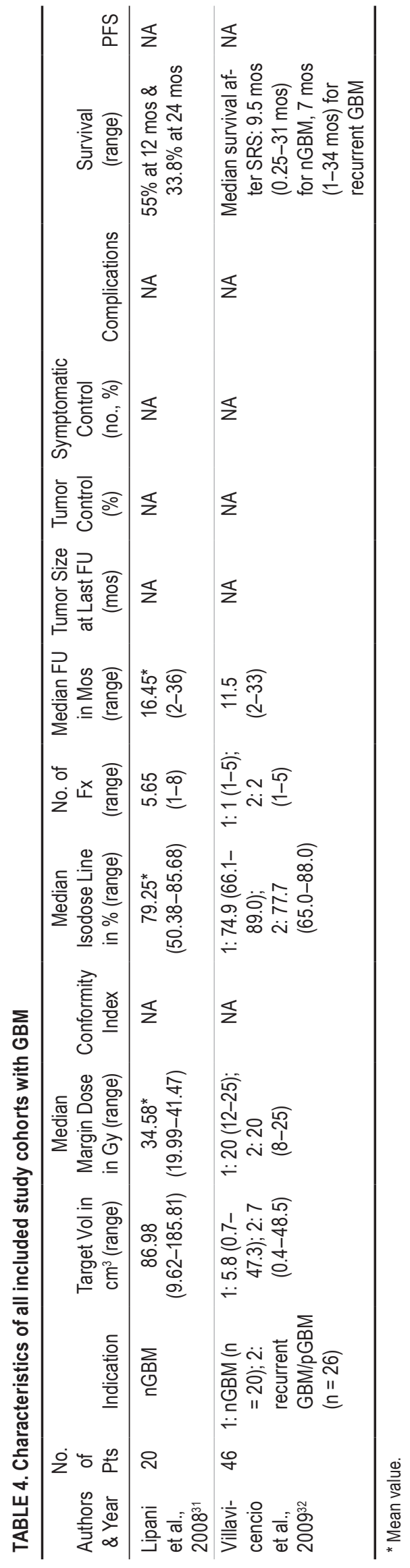

\section{Discussion}

The linear accelerator (LINAC) and Gamma Knife paved the way to a revolution in the clinical practice of neurosurgery toward a noninvasive approach. The CK has further expanded the treatment capabilities of SRS through the introduction of a mask-based setup, the implementation of a fractionated treatment schedule, and the possibility to target extracranial diseases. The neurosurgical diseases included in our analysis can be classified into three groups on the basis of the role of SRS in the management paradigm. In the first group are diseases for which SRS has a prominent and expanding role as a first-line treatment, such as brain metastases, vestibular schwannomas, and glomus jugulare tumors. In the second group are diseases for which SRS is usually adjuvant to other techniques or a second line, such as meningiomas, spine metastases, AVMs, and TN. Notably, in this group, the role of SRS as a stand-alone treatment is quickly expanding for meningiomas and over the next years could become the most frequent treatment. In the third group are diseases for which the role of SRS is still under investigation, such as GBM. Since SRS is not validated for the routine clinical management of GBM, there is not a clear trend on its use at our institution. In our recent yet limited experience, SRS has been mainly used to treat pGBM in patients who have already undergone surgery and radiochemotherapy at the initial diagnosis, according to the Stupp protocol. ${ }^{30}$ In these patients, hypofractionated SRS (40 Gy in 5 consecutive fractions) was used to treat well-demarcated, small, enhancing pGBM nodules either when a second surgery was not the patient's preferred option or when a second surgery carried significant risks due to tumor location or general clinical conditions. According to the Congress of Neurological Surgeons (CNS) guidelines published in 2014, ${ }^{39}$ regarding the role of radiation treatment for pGBM, level III evidence suggests that radiation treatment, including SRS and hypofractionated SRS, can be safely used after first-line combined multimodal treatment with chemotherapy and radiation. In this subset of patients, radiation treatment could lead to a potential, yet limited, benefit in terms of local tumor control and the patient's neurological status and quality of life before any further tumor progression.

In the CNS guidelines published in 2008, regarding the role of radiation treatment for $\mathrm{nGBM},{ }^{40}$ level I evidence suggests that SRS as a boost to EBRT is not beneficial and is not recommended in the routine management of nGBM. At our institution, in the limited context of early-stage clinical trials, hypofractionated SRS (40 Gy in 5 consecutive fractions) has been recently used as adjuvant treatment with concurrent temozolomide to treat resection cavities and enhancing nodular tumor residuals of nGBM. More than a decade ago, collaborative studies ${ }^{31,32}$ reported the use of SRS either as a primary treatment or as a radiosurgical boost shortly after surgery or surgery and standard fractionated radiotherapy, although the results in terms of overall survival did not appear superior to standard postoperative radiochemotherapy.

The classification presented above is based on a singleinstitution experience and is not necessarily generalizable to other institutions and cannot be applied for decisionmaking at a single-patient level. Another limitation to gen- 


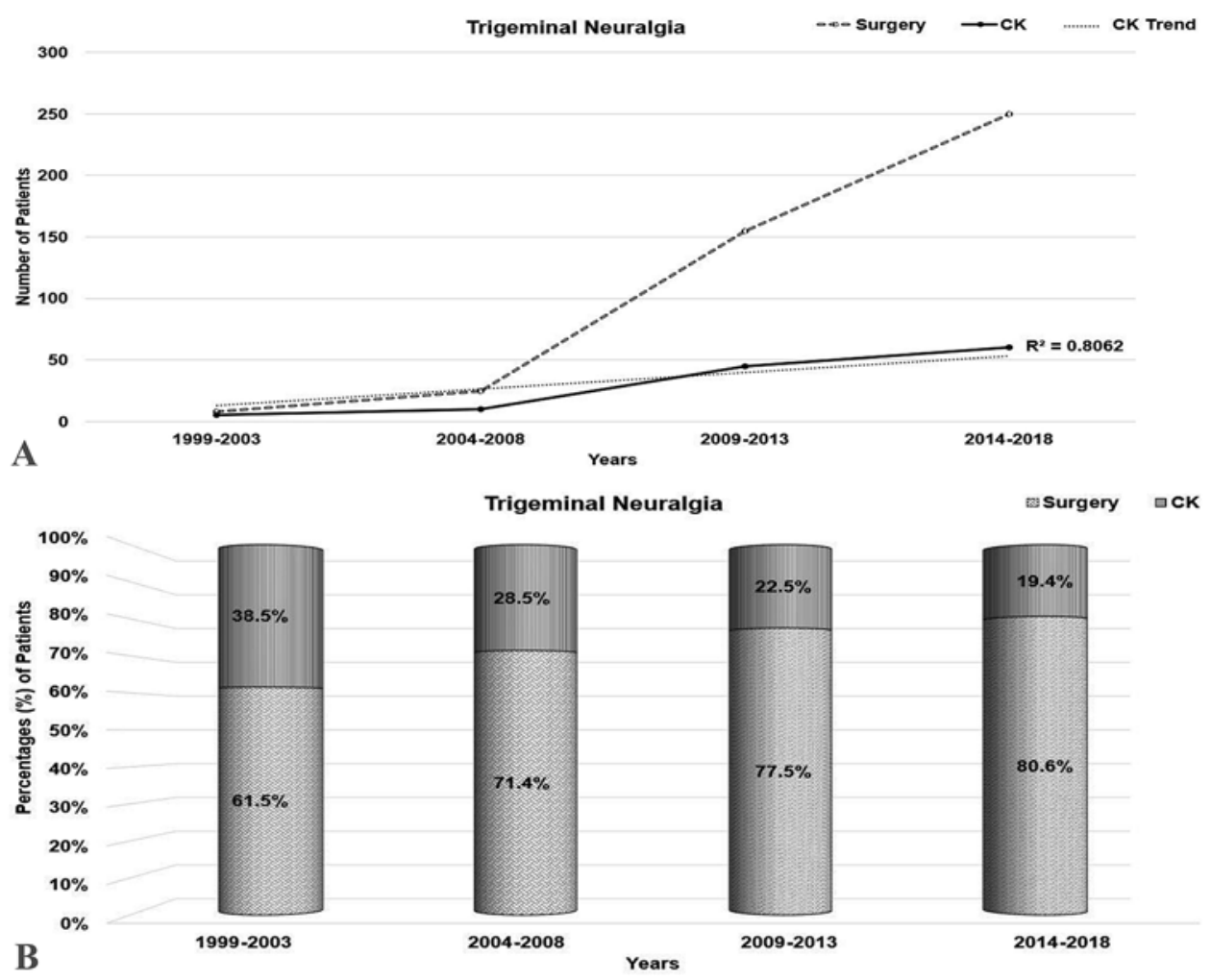

FIG. 4. A: Number of patients with TN treated with surgery or CK radiosurgery in consecutive 5-year time frames over the past 20 years (1999-2018). The CK trend line is a graphic representation of the regression coefficient $\left(R^{2}\right)$ of the cases performed with CK over the same time frames $\left(R^{2}=0.806\right)$. B: The percentage of patients treated with each treatment modality in each 5 -year period, with the most common being surgery in 2014-2018 (80.6\%). The upper portion of each column represents the percentage of patients that underwent $\mathrm{CK}$, and the lower portion represents the percentage that underwent surgery.

eralizability is the retrospective nature of the present study and the lack of outcome measures. Thus, despite the outcome data provided by our systematic review of the studies performed at our institution, the study design does not allow one to draw conclusions on the effectiveness of CK for the treatment of specific diseases. Moreover, some common treatment techniques are missing because of technical software limitations, such as EBRT for spinal metastases, novel therapeutic agents for brain metastases, and medical and ablation treatments for TN. In our analysis, several diseases, mainly those belonging to the third group, were not included despite encouraging results after SRS, such as craniopharyngiomas, pituitary tumors, pineal tumors, facetogenic back pain, and medically intractable chronic headache. In the future, CK SRS will also be applied to the treatment of neurological disorders such as movement disorders, medically refractory epilepsy, and psychiatric disorders such as obsessive-compulsive disorder and depression.

Over the past 2 decades, the CK system has undergone several software and hardware improvements, leading to more efficient treatment planning and delivery, as well as reduced treatment delivery time. Thus, progressive expansion of the indications for CK radiosurgery was possible in terms of target histology, target number, and volume.

From a software viewpoint, the treatment planning system was upgraded from On-Target to Multiplan to Precision, with better optimization algorithms introduced at each upgrade, which generated progressively more time- efficient and conformal treatment plans. The introduction of the Monte-Carlo dose calculation engine allowed for improved matching of planning doses with measured doses, particularly around air cavities. Deformable image registration and automatic segmentation have also improved efficiency in the general workflow of treatment plan generation. The Precision planning software, together with the latest VOLO optimizer, significantly reduced the amount of time required to develop treatment plans while improving the quality and efficiency of treatment. ${ }^{41}$

From a hardware viewpoint, the optimized machine path during treatment, which reduced the robotic arm travel time between the nodes when no radiation is delivered, as well as the increased LINAC dose rate from $200 \mathrm{MU} / \mathrm{min}$ to $1000 \mathrm{MU} / \mathrm{min}$, reduced treatment times. Moreover, application of the IRIS variable collimator and, more recently, the multileaf collimator has shortened the treatment time further while maintaining or improving treatment plan quality. ${ }^{42}$

\section{Conclusions}

In our experience, CK SRS emerged as an effective primary treatment for brain metastases, vestibular schwannomas, and glomus jugulare tumors. CK is mainly used as adjuvant treatment or a second-line treatment for meningiomas, spinal metastases, TN, and AVMs. CK is emerging as a palliative option for GBM and as a novel technique for 


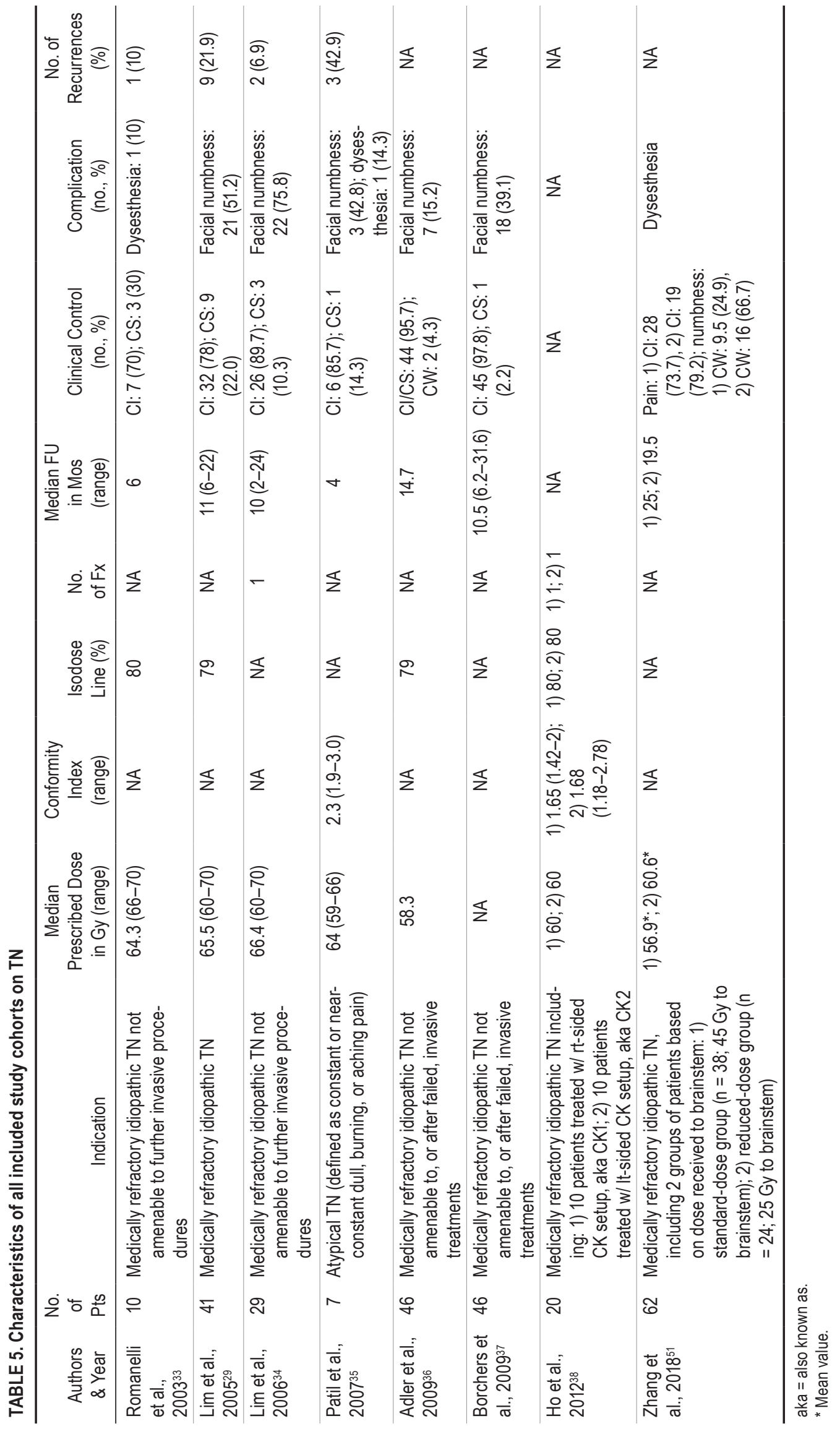


treating a myriad of neurological disorders, such as pain syndromes and neuroendocrine disorders, movement disorders, and psychiatric disorders.

\section{References}

1. Liberati A, Altman DG, Tetzlaff J, et al. The PRISMA statement for reporting systematic reviews and meta-analyses of studies that evaluate health care interventions: explanation and elaboration. J Clin Epidemiol. 2009;62(10):e1-e34.

2. Gupta R, Moore JM, Amorin A, et al. Long-term follow up data on difficult to treat intracranial arteriovenous malformations treated with the CyberKnife. J Clin Neurosci. 2019;61: 120-123.

3. Ding D, Yen CP, Starke RM, et al. Outcomes following single-session radiosurgery for high-grade intracranial arteriovenous malformations. Br J Neurosurg. 2014;28(5):666-674.

4. Sinclair J, Chang SD, Gibbs IC, Adler JR Jr. Multisession CyberKnife radiosurgery for intramedullary spinal cord arteriovenous malformations. Neurosurgery. 2006;58(6):1081-1089.

5. Sinclair J, Chang SD, Gibbs IC, Adler JR Jr. Cyber-Knife Radiosurgery for intramedullary spinal cord arteriovenous malformations. In: Mould RF, Schulz RA, eds. Robotic Radiosurgery. Vol 1. CyberKnife Society Press; 2005:187-196.

6. Kalani MA, Choudhri O, Gibbs IC, et al. Stereotactic radiosurgery for intramedullary spinal arteriovenous malformations. J Clin Neurosci. 2016;29:162-167.

7. Adler JR Jr, Gupta G, Chang SD, et al. CyberKnife ablation for intramedullary spinal cord arteriovenous malformations (AVMs): a promising new therapeutic approach. Cureus. 2010;2(8):e14.

8. Zhang M, Connolly ID, Teo MK, et al. Management of arteriovenous malformations associated with developmental venous anomalies: a literature review and report of 2 cases. World Neurosurg. 2017;106:563-569.

9. Adler JR, Cox RS. Preliminary clinical experience with the CyberKnife: image-guided stereotactic radiosurgery. In: Kondziolka D, ed. Radiosurgery 1995. Karger; 1996:316326.

10. Yamamoto M, Serizawa T, Shuto T, et al. Stereotactic radiosurgery for patients with multiple brain metastases (JLGK0901): a multi-institutional prospective observational study. Lancet Oncol. 2014;15(4):387-395.

11. Brennan C, Yang TJ, Hilden P, et al. A phase 2 trial of stereotactic radiosurgery boost after surgical resection for brain metastases. Int J Radiat Oncol Biol Phys. 2014;88(1): 130-136.

12. Hara W, Tran P, Li G, et al. CyberKnife for brain metastases of malignant melanoma and renal cell carcinoma. Neurosurgery. 2009;64(2 Suppl):A26-A32.

13. Liu SH, Murovic J, Wallach J, et al. CyberKnife radiosurgery for brainstem metastases: management and outcomes and a review of the literature. J Clin Neurosci. 2016;25:105-110.

14. Azad TD, Esparza R, Chaudhary N, Chang SD. Stereotactic radiosurgery for metastasis to the craniovertebral junction preserves spine stability and offers symptomatic relief. $J$ Neurosurg Spine. 2016;24(2):241-247.

15. Murovic J, Ding V, Han SS, et al. Impact of CyberKnife radiosurgery on median overall survival of various parameters in patients with 1-12 brain metastases. Cureus. 2017;9(12): e1926.

16. Lutz S, Berk L, Chang E, et al. Palliative radiotherapy for bone metastases: an ASTRO evidence-based guideline. Int J Radiat Oncol. 2011;79(4):965-976.

17. Gibbs IC, Kamnerdsupaphon P, Ryu MR, et al. Image-guided robotic radiosurgery for spinal metastases. Radiother Oncol. 2007;82(2):185-190.

18. Veeravagu A, Lieberson RE, Mener A, et al. CyberKnife stereotactic radiosurgery for the treatment of intramedullary spinal cord metastases. J Clin Neurosci. 2012;19(9):12731277.

19. Pham CJ, Chang SD, Gibbs IC, et al. Preliminary visual field preservation after staged CyberKnife radiosurgery for perioptic lesions. Neurosurgery. 2004;54(4):799-812.

20. Adler JR Jr, Gibbs IC, Puataweepong P, Chang SD. Visual field preservation after multisession CyberKnife radiosurgery for perioptic lesions. Neurosurgery. 2006;59(2):244-254.

21. Cheshier SH, Hanft SJ, Adler JR Jr, Chang SD. CyberKnife radiosurgery for lesions of the foramen magnum. Technol Cancer Res Treat. 2007;6(4):329-336.

22. Patil CG, Hoang S, Borchers DJ III, et al. Predictors of peritumoral edema after stereotactic radiosurgery of supratentorial meningiomas. Neurosurgery. 2008;63(3):435-442.

23. Tuniz F, Soltys SG, Choi CY, et al. Multisession CyberKnife stereotactic radiosurgery of large, benign cranial base tumors: preliminary study. Neurosurgery. 2009;65(5):898-907.

24. Choi CYH, Soltys SG, Gibbs IC, et al. Cyberknife stereotactic radiosurgery for treatment of atypical (WHO grade II) cranial meningiomas. Neurosurgery. 2010;67(5):1180-1188.

25. Fatima N, Meola A, Pollom E, et al. Stereotactic radiosurgery for large benign intracranial tumors. World Neurosurg. 2020; 134:e172-e180.

26. Hansasuta A, Choi CYH, Gibbs IC, et al. Multisession stereotactic radiosurgery for vestibular schwannomas: singleinstitution experience with 383 cases. Neurosurgery. 2011; 69(6):1200-1209.

27. Lim M, Gibbs IC, Adler JR Jr, et al. The efficacy of linear accelerator stereotactic radiosurgery in treating glomus jugulare tumors. Technol Cancer Res Treat. 2003;2(3):261-265.

28. Lim M, Gibbs IC, Adler JR Jr, Chang SD. Efficacy and safety of stereotactic radiosurgery for glomus jugulare tumors. $\mathrm{Neu}$ rosurg Focus. 2004;17(2):E11.

29. Lim M, Villavicencio AT, Burneikiene S, et al. CyberKnife radiosurgery for idiopathic trigeminal neuralgia. Neurosurg Focus. 2005;18(5):E9.

30. Stupp R, Mason WP, van den Bent MJ, et al. Radiotherapy plus concomitant and adjuvant temozolomide for glioblastoma. N Engl J Med. 2005;352(10):987-996.

31. Lipani JD, Jackson PS, Soltys SG, et al. Survival following CyberKnife radiosurgery and hypofractionated radiotherapy for newly diagnosed glioblastoma multiforme. Technol Cancer Res Treat. 2008;7(3):249-255.

32. Villavicencio AT, Burneikienė S, Romanelli P, et al. Survival following stereotactic radiosurgery for newly diagnosed and recurrent glioblastoma multiforme: a multicenter experience. Neurosurg Rev. 2009;32(4):417-424.

33. Romanelli P, Heit G, Chang SD, et al. Cyberknife radiosurgery for trigeminal neuralgia. Stereotact Funct Neurosurg. 2003;81(1-4):105-109.

34. Lim M, Cotrutz C, Romanelli P, et al. Stereotactic radiosurgery using CT cisternography and non-isocentric planning for the treatment of trigeminal neuralgia. Comput Aided Surg. 2006;11(1):11-20.

35. Patil CG, Veeravagu A, Bower RS, et al. CyberKnife radiosurgical rhizotomy for the treatment of atypical trigeminal nerve pain. Neurosurg Focus. 2007;23(6):E9.

36. Adler JR, Bower R, Gupta G, et al. Nonisocentric radiosurgical rhizotomy for trigeminal neuralgia. Neurosurgery. 2009; 64(suppl 2):84-90.

37. Borchers JD III, Yang H-J, Sakamoto GT, et al. CyberKnife stereotactic radiosurgical rhizotomy for trigeminal neuralgia: anatomic and morphological considerations. Neurosurgery. 2009;64(2 Suppl):A91-A95.

38. Ho A, Lo AT, Dieterich $\mathrm{S}$, et al. Trigeminal neuralgia treatment dosimetry of the Cyberknife. Med Dosim. 2012;37(1): 42-46.

39. Ryu S, Buatti JM, Morris A, et al. The role of radiotherapy in the management of progressive glioblastoma: a systematic 
review and evidence-based clinical practice guideline. $J \mathrm{Neu}$ rooncol. 2014;118(3):489-499.

40. Buatti J, Ryken TC, Smith MC, et al. Radiation therapy of pathologically confirmed newly diagnosed glioblastoma in adults. J Neurooncol. 2008;89(3):313-337.

41. Schüler E, Lo A, Chuang CF, et al. Clinical impact of the VOLO optimizer on treatment plan quality and clinical treatment efficiency for CyberKnife. J Appl Clin Med Phys. 2020; 21(5):38-47.

42. McGuinness CM, Gottschalk AR, Lessard E, et al. Investigating the clinical advantages of a robotic LINAC equipped with a multileaf collimator in the treatment of brain and prostate cancer patients. J Appl Clin Med Phys. 2015;16(5): 284-295.

43. Gibbs IC, Patil C, Gerszten PC, et al. Delayed radiationinduced myelopathy after spinal radiosurgery. Neurosurgery. 2009;64(suppl 2):67-72.

44. Soltys SG, Adler JR, Lipani JD, et al. Stereotactic radiosurgery of the postoperative resection cavity for brain metastases. Int J Radiat Oncol Biol Phys. 2008;70(1):187-193.

45. Lieberson RE, Veeravagu A, Eckermann JM, et al. Intramedullary spinal cord metastasis from prostate carcinoma: a case report. J Med Case Rep. 2012;6(1):139.

46. Atalar B, Modlin LA, Choi CYH, et al. Risk of leptomeningeal disease in patients treated with stereotactic radiosurgery targeting the postoperative resection cavity for brain metastases. Int J Radiat Oncol Biol Phys. 2013;87(4):713-718.

47. Lim M, Bower R, Nangiana JS, et al. Radiosurgery for glomus jugulare tumors. Technol Cancer Res Treat. 2007;6(5): 419-423.

48. Chang SD, Gibbs IC, Sakamoto GT, et al. Staged stereotactic irradiation for acoustic neuroma. Neurosurgery. 2005;56(6): $1254-1263$.

49. Dodd RL, Ryu MR, Kamnerdsupaphon P, et al. CyberKnife radiosurgery for benign intradural extramedullary spinal tumors. Neurosurgery. 2006;58(4):674-685.

50. Teo M, Zhang M, Li A, et al. The outcome of hypofractionated stereotactic radiosurgery for large vestibular schwannomas. World Neurosurg. 2016;93:398-409.

51. Zhang M, Lamsam LA, Schoen MK, et al. Brainstem dose constraints in nonisometric radiosurgical treatment plan- ning of trigeminal neuralgia: a single-institution experience. World Neurosurg. 2018;113:e399-e407.

\section{Disclosures}

Dr. Gibbs receives honoraria from Accuray Inc. Dr. Adler is an employee of Zap Surgical Systems.

\section{Author Contributions}

Conception and design: Meola. Acquisition of data: Fatima, Chuang, Shahsavari. Analysis and interpretation of data: Meola, Fatima, Ding, Pollom, Soltys, Chuang. Drafting the article: Meola, Fatima. Critically revising the article: Meola, Fatima, Pollom, Soltys, Chuang, Shahsavari, Hancock, Gibbs, Adler, Chang. Reviewed submitted version of manuscript: Meola, Fatima, Pollom, Soltys, Chuang, Shahsavari, Gibbs, Adler. Approved the final version of the manuscript on behalf of all authors: Meola. Statistical analysis: Fatima, Ding. Administrative/technical/ material support: Meola, Ding, Chang. Study supervision: Meola, Chang.

\section{Supplemental Information}

\section{Online-Only Content}

Supplemental material is available with the online version of the article.

Supplemental Tables. https://thejns.org/doi/suppl/10.3171/ 2020.9.JNS201484.

\section{Previous Presentations}

Portions of this work were presented at the virtual 2020 American Association of Neurological Surgeons Annual Scientific Meeting.

\section{Correspondence}

Antonio Meola: Stanford University School of Medicine, Stanford,CA. ameola@stanford.edu. 